\title{
Transient bunch compression using pulsed phase modulation in high-energy electron storage rings
}

\author{
Glenn Decker, Michael Borland, Doug Horan, Alex Lumpkin, Nicholas Sereno, and Bingxin Yang \\ Advanced Photon Source, Argonne National Laboratory, 9700 South Cass Avenue, Argonne, Illinois 60439, USA \\ Samuel Krinsky \\ National Synchrotron Light Source, Brookhaven National Laboratory, P.O. Box 5000, Upton, New York, 11973, USA
}

(Received 18 January 2006; published 13 December 2006)

\begin{abstract}
A method for producing short electron bunches in an electron storage ring using pulsed phase modulation has been demonstrated. A simple theoretical model was validated using the particle tracking code ELEGANT, and the bunch compression process was observed experimentally in the Advanced Photon Source storage ring using a visible light streak camera. Compression to $54 \%$ of the initial bunch length was achieved.
\end{abstract}

DOI: 10.1103/PhysRevSTAB.9.120702

PACS numbers: 41.75.Lx, 41.60.-m, 41.85.Ew

\section{INTRODUCTION}

A number of synchrotron radiation x-ray experiments at the Advanced Photon Source make use of the fast time structure of the particle beam, and would benefit further from even shorter bunches than those normally available during machine operation. Efforts to produce very short pulses in new free-electron laser projects such as the Linac Coherent Light Source [1] and the Subpicosecond Photon Pulse Facility [2] at Stanford, the European X-ray FreeElectron Laser Project [3], and energy recovery linacs [4] at Jefferson Laboratory and Cornell are further evidence of the high level of interest in this area.

The steady-state bunch length in electron storage rings is determined in general by a combination of quantum excitation and bunch-current-dependent effects such as potential well distortion and the microwave instability. At the Advanced Photon Source (APS), this results in significant bunch lengthening as the single bunch current is increased. For this reason, efforts to reduce the equilibrium bunch length by means of a momentum compaction reduction, for example, have been successful only in the case of very small bunch current [5,6]. A number of schemes have been put forward to overcome bunch lengthening and provide very short $\mathrm{x}$-ray pulses $[7,8]$. The technique described here makes use of rf phase modulation to excite a quadrupole bunch shape oscillation in a controlled fashion, while maintaining high peak current and low emittance. Considerable effort is sometimes required to suppress oscillations of this type, which result from undesirable beam instabilities [9,10]. Amplitude modulation [11,12] and phase modulation [13-16] have been used in electron storage rings, both to improve beam lifetime by lengthening the bunch to overcome Touschek scattering, and to cure instabilities. A recent experiment demonstrated bunch compression in an electron storage ring using amplitude modulation [17].
Detailed experimental studies of the effects of rf phase modulation on particle motion in an electron storage ring [18] and a proton cyclotron [19] have been performed. Amplitude modulation [20-23] and bunch compression techniques [24] in proton machines bear the closest resemblance to the method described here. Since the synchronous phase in an electron storage ring varies strongly with rf voltage, amplitude modulation results in undesirable centroid oscillations, in addition to a shape oscillation. In a proton ring, the technique described here is analogous to attempting the phase-space manipulations while the beam energy is rapidly ramped. Phase modulation of the total $\mathrm{rf}$ voltage in an electron ring also causes complicated phasespace motion, especially for the large levels of modulation we require (up to 45 degrees $p-p$ ).

The APS rf cavities are normally arranged to be driven from two separate klystrons, each unit powering a set of eight cavities. In the simplest conception, one can introduce an interstation phase difference between the two systems until the particle beam is aligned at the zerocrossing phase of one of them. This effectively separates the focusing and acceleration functions of the rf. The system at zero crossing behaves like a fixed energy proton ring's rf system, performing only focusing, while the second system provides the energy recovery function. If the voltage of this second system is set equal to the energy loss per turn $U_{0}$, it will be on-crest, performing acceleration but no focusing, and the two systems will be in quadrature.

Amplitude modulation of the zero-crossing system at twice the synchrotron frequency $f_{s}$ will result in the desired quadrupole bunch shape oscillation, in a fashion nearly identical to that described by Bai et al. [21]. The mechanism behind the induced oscillation is that of a driven parametric oscillator, with the parameter in question being the synchrotron frequency. Rather than using amplitude modulation of the zero-crossing system, a phase modulation of the on-crest system will produce a large 
synchrotron tune modulation with minimal amplitude modulation, resulting in the desired shape oscillation. It is this technique that is the topic of our investigations.

Experiments conducted at the APS using this technique have demonstrated a factor of 2 reduction in bunch length. The mechanisms that limit the technique to a factor of 2 for the special case of the APS are not immediately obvious, and it is likely that compression by larger factors is possible for different machines and operating conditions. The methodology developed here provides a general framework for quantitatively determining the effectiveness of the method. The primary thrust of this article is the experimental demonstration of the compression technique, while preserving both bunch intensity and emittance. The authors are aware of no other means for accomplishing this short of the introduction of major machine modifications such as the installation of crab cavities to implement the Zholents x-ray pulse compression scheme [7].

A simple theoretical model of the parametric resonance and resulting shape oscillation is given in Sec. II. An approximate solution to the resulting differential equation is contrasted with an exact numerical solution. Simulation of the longitudinal dynamics including the phase modulation is described in Sec. III, and comparisons are made with the simple theoretical model. A discussion of energy spread effects is given in Sec. IV, and in Sec. V an experiment conducted on the APS storage ring is described, and streak camera results are given. The results are compared in detail with particle tracking.

\section{PARAMETRIC RESONANCE GROWTH RATE}

Consider the longitudinal equation of motion for a particle undergoing small amplitude synchrotron oscillations in the absence of synchrotron radiation damping:

$$
\frac{d^{2} \phi}{d t^{2}}+\Omega_{S}^{2} \phi=0
$$

where $\phi=\psi-\psi_{S}$, with $\psi=\omega_{\text {rf }} t$, i.e., $\phi$ is the particle's time coordinate written in units of rf phase measured relative to the synchronous phase $\psi_{S}$. The angular synchrotron frequency $\Omega_{S}=2 \pi f_{S}$ is given by

$$
\Omega_{s}^{2}=\left.\frac{-\omega_{\mathrm{rf}} \alpha_{C} e}{E T_{0}} \frac{d V}{d \psi}\right|_{\psi=\psi_{s}} .
$$

Here the notation follows that of Wiedemann [25], slightly modified for relativistic electrons. Also, $\omega_{\mathrm{rf}}$ is $2 \pi$ times the $\mathrm{rf}$ frequency $f_{\mathrm{rf}}, \alpha_{C}$ is the momentum compaction, $e$ is the electronic charge, $E$ is the energy, $T_{0}$ is the revolution period, and $V(\psi)$ is the rf voltage waveform. For the general case of two rf systems with the same frequency but independent amplitudes and phases,

$$
V(\psi)=V_{1} \sin \left(\psi-\psi_{1}\right)+V_{2} \sin \left(\psi-\psi_{2}\right)
$$

Declaring the system with voltage $V_{2}$ to be the on-crest system, a constraint is imposed by requiring that the energy gained by particles at the synchronous phase $\psi_{S}$ be equal to the synchrotron radiation loss per turn $U_{0}$ :

$$
\begin{aligned}
U_{0} & =V_{1} \sin \left(\psi_{S}-\psi_{1}\right)+V_{2} \sin \left(\psi_{S}-\psi_{2}\right) \\
& =V_{1} \sin \left(\psi_{S}-\psi_{1}\right)+V_{2},
\end{aligned}
$$

since $\psi_{S}-\psi_{2}=\pi / 2$ when no modulation is applied. To include the phase modulation, the quantity $\psi-\psi_{2}$ in Eq. (3) should be replaced by

$$
\psi-\psi_{2}=\pi / 2+\Delta \phi \cos \left(\omega_{m} t\right),
$$

where $\Delta \phi$ is the phase-modulation amplitude and $\omega_{m}$ is the modulation frequency.

Combining Eqs. (3)-(5), and assuming that the phasemodulation amplitude $\Delta \phi$ is small compared to unity, the angular synchrotron frequency becomes

$$
\Omega_{s}^{2}=\frac{\omega_{R F} \alpha_{C} e}{E T_{0}}\left[V_{0}-V_{2} \Delta \phi \cos \left(\omega_{m} t\right)\right],
$$

where the quantity $V_{0}$ is given by

$$
V_{0}=\sqrt{V_{1}^{2}-\left(U_{0}-V_{2}\right)^{2}}
$$

Here the effects of the two systems are seen explicitly, especially if $U_{0}=V_{2}$, so that $V_{0}=V_{1}$, and the two systems are in quadrature. In this case, the nominal synchrotron tune is set by $V_{1}$ alone. For the on-crest system, the voltage $V_{2}$ provides tune modulation in addition to energy recovery. Finally, the equation of motion can be written

$$
\frac{d^{2} \phi}{d t^{2}}+\Omega_{s 0}^{2}\left[1-2 m \cos \left(\omega_{m} t\right)\right] \phi=0,
$$

where

$$
\Omega_{s 0}^{2}=\frac{\omega_{\mathrm{rf}} \alpha_{C} e}{E T_{0}} V_{0}=\frac{\omega_{\mathrm{rf}} \alpha_{C} e}{E T_{0}} \sqrt{V_{1}^{2}-\left(U_{0}-V_{2}\right)^{2}},
$$

and the modulation parameter $m$ is given by

$$
m=\frac{\omega_{\mathrm{rf}} \alpha_{C} e V_{2}}{2 \Omega^{2}{ }_{s 0} E T_{0}} \Delta \phi=\frac{V_{2}}{2 V_{0}} \Delta \phi .
$$

Equation (8) is an instance of Mathieu's equation. Fortunately, this equation is well documented, and some excellent approximate solutions are available [26]. The principal resonance occurs when $\omega_{m}=2 \Omega_{s 0}$. In this case, the solution to Eq. (8) is well approximated by

$$
\begin{aligned}
\phi(t) \approx & \frac{1}{2}\left(\phi_{0}-\dot{\phi}_{0} / \Omega_{s 0}\right) e^{\alpha t}\left(\cos \Omega_{s 0} t-\sin \Omega_{s 0} t\right) \\
& +\frac{1}{2}\left(\phi_{0}+\dot{\phi}_{0} / \Omega_{s 0}\right) e^{-\alpha t}\left(\cos \Omega_{s 0} t+\sin \Omega_{s 0} t\right),
\end{aligned}
$$

where the instability growth rate $\alpha$ is given by

$$
\alpha=\frac{m \Omega_{s 0}}{2}=\frac{\omega_{\mathrm{rf}} \alpha_{C} e V_{2}}{4 \Omega_{s 0} E T_{0}} \Delta \phi .
$$

For the quadrature condition $V_{1}=V_{2}=U_{0}$, the growth 
rate becomes

$$
V_{1}=V_{2}=U_{0} \Rightarrow \alpha_{q}=\frac{\Omega_{s 0}}{4} \Delta \phi
$$

The essential thing to notice about Eq. (11) is that the locations of the zero crossings become independent of the initial conditions for times $t \gg 1 / \alpha$. What this implies is that in the case of a perfectly linear phase space, the bunch length will eventually shrink to zero at each of the times corresponding to those zero crossings, which occur twice each synchrotron period. To demonstrate this, Eq. (11) can be rewritten as

$$
\phi(t) \approx \phi_{0} C(t)+\left(\dot{\phi}_{0} / \Omega_{s 0}\right) S(t) .
$$

For an ensemble of particles with a given distribution of initial phase-space parameters $\left\{\phi_{0 k}, \dot{\phi}_{0 k}\right\}$, the evolution of the mean square phase can be written as

$$
\left\langle\phi^{2}(t)\right\rangle \approx\left\langle\phi_{0}^{2}\right\rangle C^{2}(t)+\left(\left\langle\dot{\phi}_{0}^{2}\right\rangle / \Omega_{s 0}^{2}\right) S^{2}(t) .
$$

The initial distribution is assumed to be symmetric, i.e., $\left\langle\phi_{0}\right\rangle=\left\langle\dot{\phi}_{0}\right\rangle=0$, and uncorrelated, $\left\langle\phi_{0} \dot{\phi}_{0}\right\rangle=0$. The result is

$$
\begin{aligned}
\left\langle\phi^{2}(t)\right\rangle \approx & \frac{1}{2}\left(\left\langle\phi_{0}^{2}\right\rangle+\left\langle\dot{\phi}_{0}^{2}\right\rangle / \Omega_{s 0}^{2}\right)\left[e^{2 \alpha t} \cos ^{2}\left(\Omega_{s 0} t+\pi / 4\right)\right. \\
& \left.+e^{-2 \alpha t} \sin ^{2}\left(\Omega_{s 0} t+\pi / 4\right)\right] \\
& +\left(\left\langle\phi_{0}^{2}\right\rangle-\left\langle\dot{\phi}_{0}^{2}\right\rangle / \Omega_{s 0}^{2}\right)\left[\cos \left(\Omega_{s 0} t+\pi / 4\right)\right. \\
& \left.\times \sin \left(\Omega_{s 0} t+\pi / 4\right)\right] .
\end{aligned}
$$

Inspection of Eq. (16) shows that the minimum bunch length, proportional to the root mean square phase, occurs twice each synchrotron period, at times $\Omega_{s 0} t=$ $(n+1 / 4) \pi$, i.e., whenever the cosine term is zero. At these times,

$$
\begin{aligned}
& \sqrt{\left\langle\phi^{2}(t)\right\rangle_{\min }} \approx \sqrt{\left(\left\langle\phi_{0}^{2}\right\rangle+\left\langle\dot{\phi}_{0}^{2}\right\rangle / \Omega_{s 0}^{2}\right) / 2} e^{-\alpha t}
\end{aligned}
$$

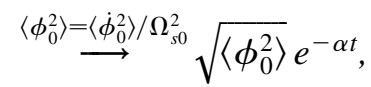

showing the exponential reduction of the bunch length with time. The second expression corresponds to a "matched" initial phase-space condition. It is easy to see that the maximum bunch length occurring at times $\Omega_{s 0} t=(n-$ $1 / 4) \pi$ grows exponentially with the same rate $\alpha$. Rewriting Eq. (16) using the matching condition $\left\langle\phi_{0}^{2}\right\rangle=$ $\left\langle\dot{\phi}_{0}^{2}\right\rangle / \Omega_{s 0}^{2}$ results in

$$
\begin{aligned}
\frac{\sigma^{2}(t)}{\sigma^{2}(0)}= & \frac{\left\langle\phi^{2}(t)\right\rangle}{\left\langle\phi^{2}(0)\right\rangle} \approx\left[e^{2 \alpha t} \cos ^{2}\left(\Omega_{s 0} t+\pi / 4\right)\right. \\
& \left.+e^{-2 \alpha t} \sin ^{2}\left(\Omega_{s 0} t+\pi / 4\right)\right] .
\end{aligned}
$$

The bunch longitudinal shape oscillations described by Eq. (18) are shown in Fig. 1. Plotted in this way, the maxima and minima in Fig. 1 lie along straight lines

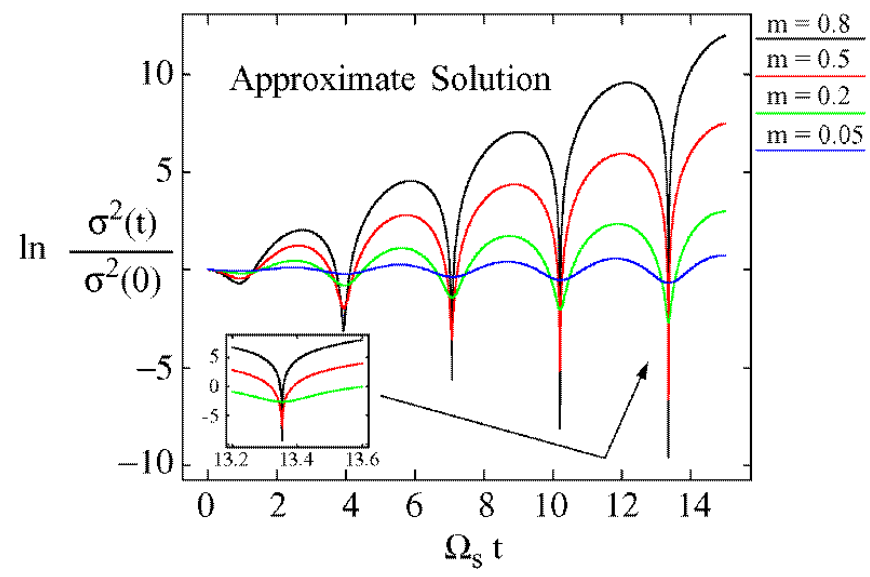

FIG. 1. (Color) .Evolution of bunch length with time: Eq. (18).

with slopes $\pm m$, the modulation parameter. The essential property of any exact solution to the original differential equation (8) is that it becomes phase locked to the phasemodulation drive signal at times $t \gg 1 / \alpha$. This ultimately results in the exponential decay of bunch length with time as in Eq. (17), and as shown graphically in Fig. 2. The functions $C(t)$ and $S(t)$ were computed numerically from the original differential equation (8), and the results were inserted into Eq. (15) to arrive at the result shown in Fig. 2.

The minimum bunch length values shown in Fig. 2 decay exponentially with nearly the same decay rate as for the approximate solutions of Fig. 1. A series of linear least squares fits to these minima were made, and shown in Fig. 3 are the slopes of the fitted lines as a function of the parameter $m$, from which the effective decay rate $\alpha$ can be determined. The error made by using the approximation $\alpha=m \Omega_{s 0} / 2$ is seen to be below $10 \%$, even for very strong phase modulation.

A number of effects have been neglected in the simple theoretical model just described. For example, synchrotron radiation damping and quantum excitation have both been ignored. It might be expected that the normal balance

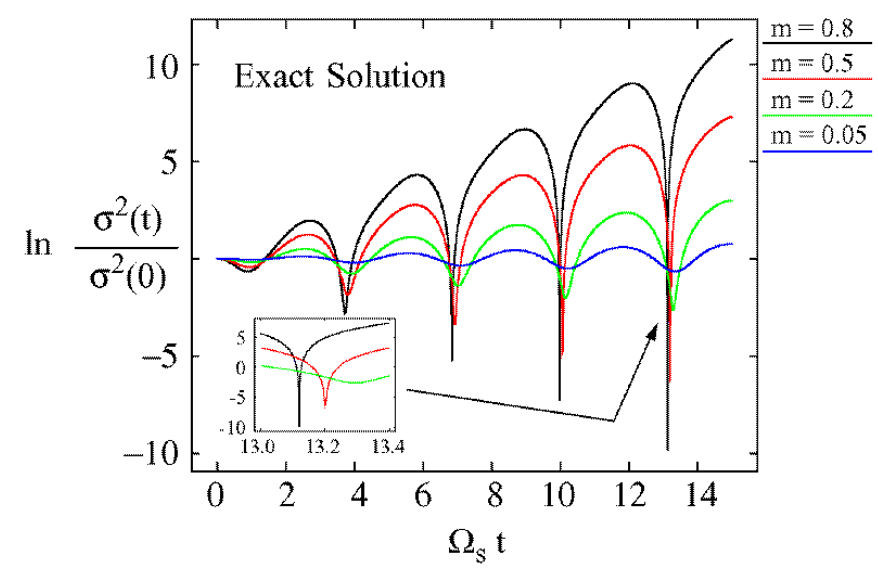

FIG. 2. (Color) Evolution of bunch length with time: numerical solution of Eq. (8). 

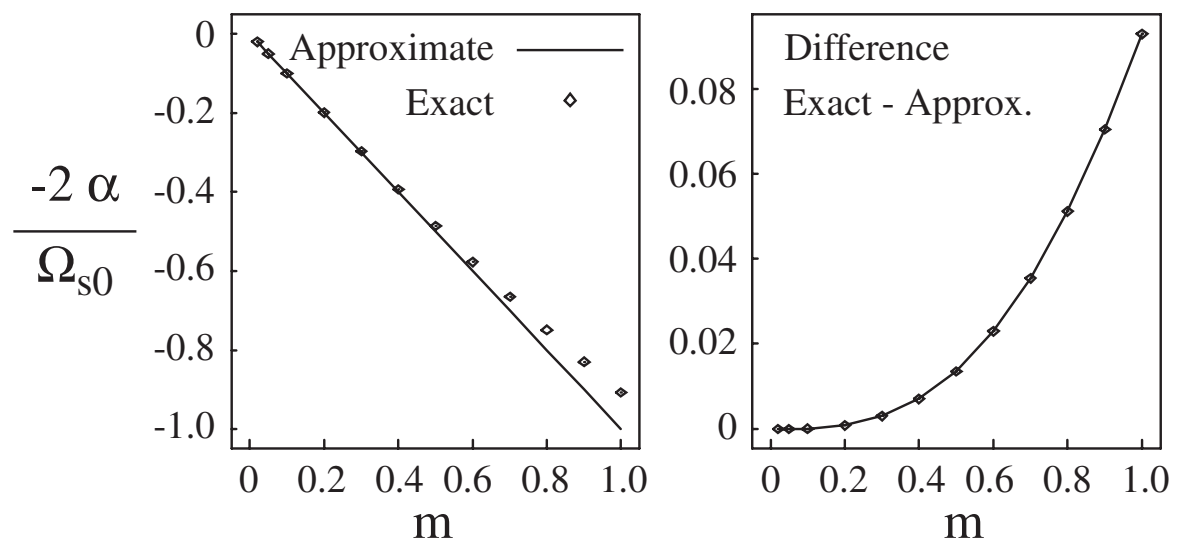

FIG. 3. Results of linear fits determining effective bunch length decay rate $\alpha$.

between these two effects, which determines the equilibrium zero-current bunch length, will be preserved. Another limitation on this model arises from the nonlinearity of longitudinal phase space. Because particles with larger amplitude will have different synchrotron periods, the compression process will be self-limiting. To investigate these effects quantitatively, it is necessary to use particle tracking simulations.

\section{SIMULATION}

The accelerator tracking code ELEGANT [27] was used to model the longitudinal dynamics of the Advanced Photon Source storage ring with the addition of the abovedescribed phase modulation. Shown in Table I are the relevant longitudinal ring parameters. Two 1-MW klystrons are normally used to drive $16 \mathrm{rf}$ cavities at $352 \mathrm{MHz}$. While the total $\mathrm{rf}$ voltage is nominally 9.4 MV, the system has been designed to operate in this mode with as much as $12 \mathrm{MV}$ total. Increasing the total $\mathrm{rf}$ voltage from 9.4 to $10.74=2 \times U_{0}$ allows the quadrature condition $V_{1}=V_{2}=U_{0}$ from Eq. (13) to be satisfied.

The program ELEGANT and the self-describing data set toolkit suite of $\mathrm{C}$ programs provide a very flexible environment for running accelerator tracking simulations using

TABLE I. Nominal APS longitudinal phase-space parameters.

\begin{tabular}{lc}
\hline \hline Energy & $7 \mathrm{GeV}$ \\
rf frequency $f_{\mathrm{rf}}$ & $351.93 \mathrm{MHz}$ \\
rf voltage & $9.4 \mathrm{MV}$ \\
Energy loss/turn $U_{0}$ & $5.37 \mathrm{MeV}$ \\
Harmonic number & 1296 \\
Synchrotron frequency $f_{S}$ & $2.16 \mathrm{kHz}$ \\
Zero-current bunch length & $19.6 \mathrm{ps} \mathrm{rms}$ \\
Energy spread $\sigma_{\delta}$ & $0.096 \% \mathrm{rms}$ \\
Energy acceptance $\varepsilon_{\text {acc }}$ & $2.5 \%$ \\
Damping time $\tau_{E}$ & $4.75 \mathrm{~ms}$ \\
Momentum compaction $\alpha_{C}$ & $2.79 \times 10^{-4}$ \\
Synchronous phase $\psi_{S}$ & $145 \mathrm{degrees}$ \\
\hline \hline
\end{tabular}

high-level scripting languages. In this case, Tcl/Tk scripts were used to configure the input files and command line options for a series of tracking runs that were submitted to a cluster of dedicated Linux computers used for accelerator studies. The tracking runs proceeded in a two-step process. Initially ELEGANT was used to generate a file containing the initial linear lattice functions. This was followed by a special step to insert longitudinal parameters, rf voltages, bunch length, etc., taking into account the phase separation between the two rf systems. Once the initial sixdimensional lattice functions were determined, a separate file was created containing the phase-modulation waveform. Finally, ELEGANT was executed a second time, making heavy use of macro substitutions to start the tracking runs with the previously determined matched phasespace parameters. Of particular importance were the "RAMPRF" lattice element used to apply the phase modulation and the "bunched beam" command, which was used to generate the initial six-dimensional particle distribution.

Shown in Fig. 4 is a typical phase-modulation waveform. A tone burst was modeled in order to limit the size of the resulting oscillations, since it was intended to apply this experimentally in a periodic fashion without losing too many particles. A hold-off period prior to the beginning of the modulation was used as a check to ensure a good initial match with no shape oscillations. The modulation frequency, equal to twice the synchrotron frequency, is $3.6 \mathrm{kHz}$ using Eq. (9). In spite of operation with higher $\mathrm{rf}$ voltage $(10.74 \mathrm{MV})$, the synchrotron frequency is lower than indicated in Table I as a result of the $90^{\circ}$ initial phase separation between the two rf systems. A total of 10000 particles were tracked through 2000 turns, amounting to 7.4 milliseconds. Each simulation required approximately two hours to complete.

The model included both quantum excitation and radiation damping. Two methods are available for modeling these synchrotron radiation effects. The first method incorporates quantum fluctuations for each magnetic element individually, and is very computationally intensive. The second method models this in a lumped fashion, with 


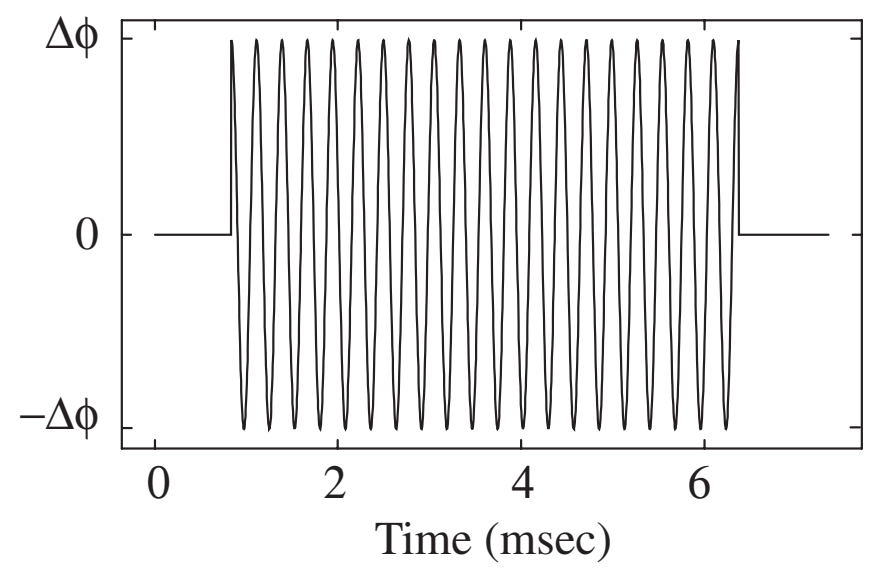

FIG. 4. Typical phase-modulation waveform used for tracking. fluctuations and damping introduced once per turn. Comparing the two methods, no significant differences were found impacting the bunch compression process, so the simpler method was used for the majority of the tracking runs.

Shown in Fig. 5 are results of the tracking runs for phase-modulation amplitudes $\Delta \phi=2.3,5.7,11.5$, and 22.9 degrees, corresponding to $m=0.02,0.05,0.1$, and 0.2 , respectively, using Eq. (10). The phase-modulation waveform shown in Fig. 4 was used here.

On the left is the rms bunch length while the right-hand plot shows rms fractional energy spread in percent. The initial bunch length, $23.5 \mathrm{ps}$ rms, is somewhat larger than indicated in Table I as a result of the DC phase separation between the two rf systems. In this configuration, the energy acceptance is $1.7 \%$, slightly smaller than indicated

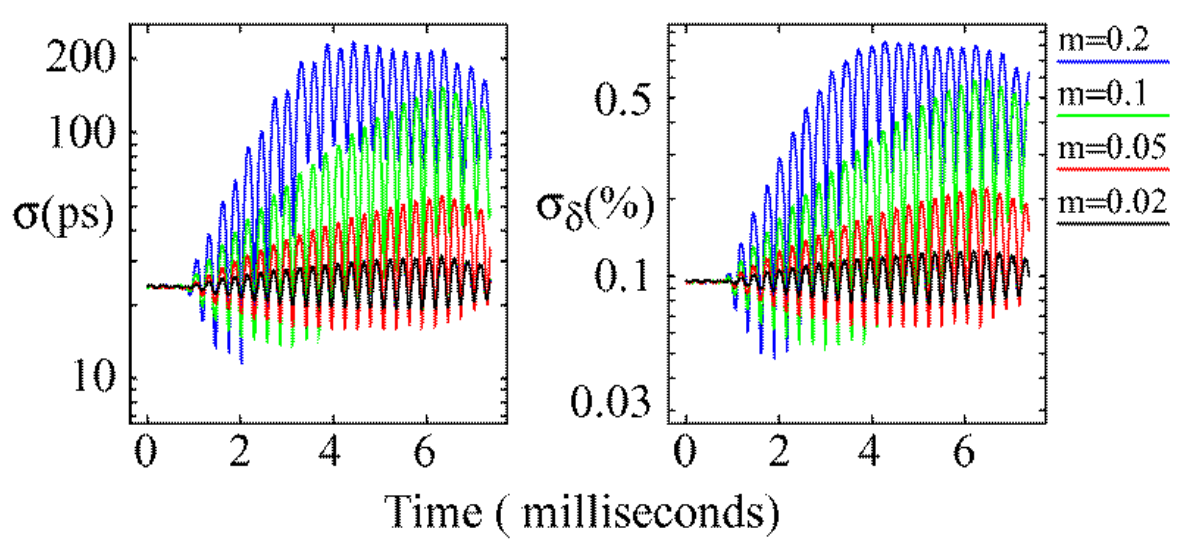

FIG. 5. (Color) Phase-modulation bunch compression technique simulation results.
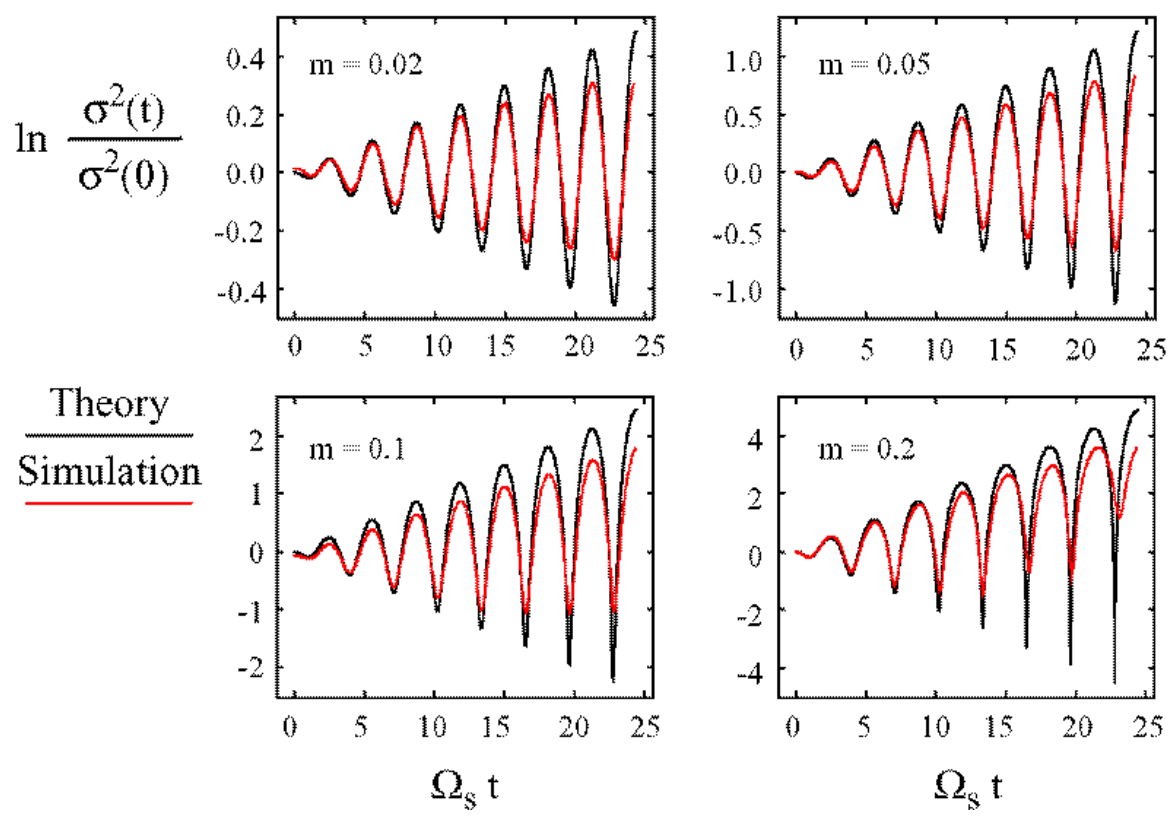

FIG. 6. (Color) Comparison between theoretical result [Eq. (18)] and simulation. 

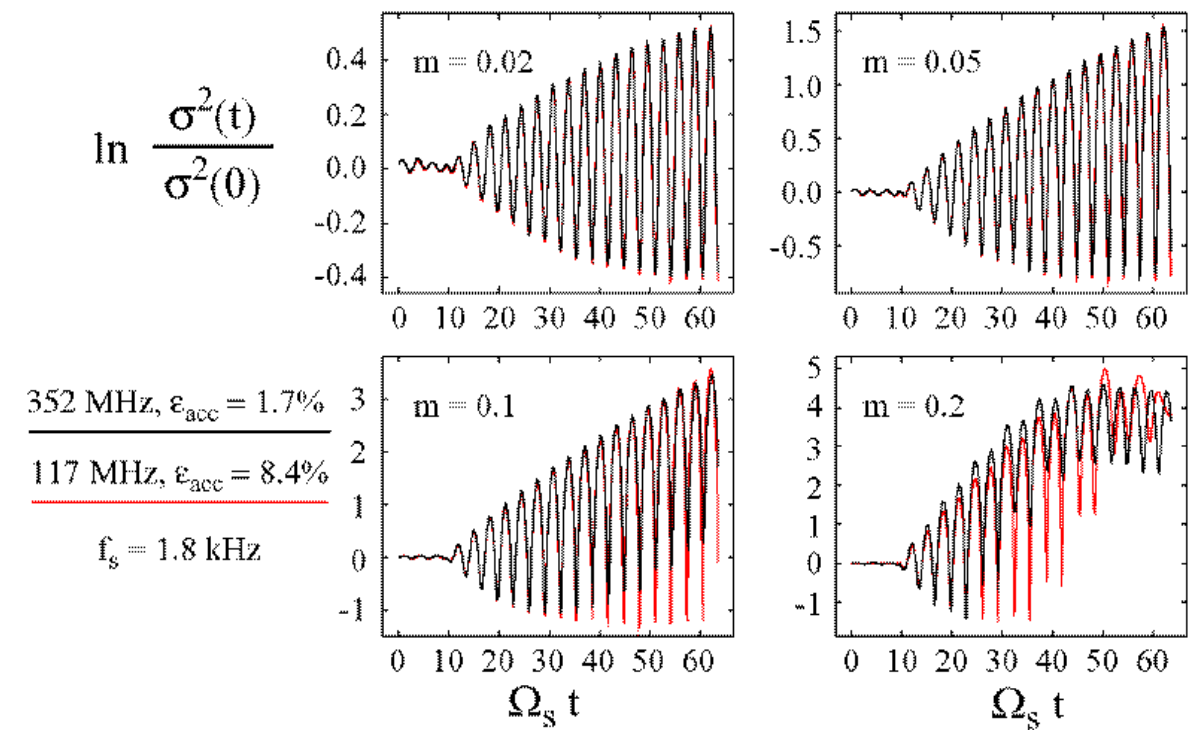

FIG. 7. (Color) Effect of energy acceptance $\varepsilon_{\text {acc }}$ on bunch compression process (simulation).

in the table. The minimum bunch length, which occurs for $m=0.2$, is $11.6 \mathrm{ps}$, a reduction of more than a factor of 2 . After an extended period the compression process breaks down, most likely as a consequence of bucket nonlinearity, quantum excitation, or some combination of the two.

Figure 6 shows a comparison between the simulation results of Fig. 5 and the analytical result [Eq. (18)], focusing on the first few cycles where the greatest bunch compression is observed. The axes in this case have been scaled for direct comparison with Figs. 1 and 2. The initial growth/decay rates from simulation are smaller in magnitude than expected from theory.

To investigate further the impact of bucket nonlinearity, a series of tracking runs were conducted with the rf frequency reduced by a factor of 3 , the voltage $V_{1}$ (the zerocrossing system) increased by a factor of 3 , and using 3 times the phase-modulation amplitude $\Delta \phi$. This has the primary effect of increasing the energy acceptance from $1.7 \%$ to $8.4 \%$ while holding the synchrotron frequency $f_{s}$ and modulation factor $m$ constant. Shown in Fig. 7 is a comparison between pairs of simulations, with the primary variable being the energy acceptance. Several modulation cycles are required to drive particles to amplitudes large enough for bucket nonlinearity to show a significant effect, visible only with strong modulation $m=0.1$ and $m=0.2$.

The discrepancy between theory and simulation seen in Fig. 6 is apparently the result of a limiting process other than bucket nonlinearity. Figure 8 shows a simulation result for an extended period using very weak phase modulation $(m=0.02)$. In this case, the simulation was allowed to run for 5000 turns, and the theoretical result is overlaid to indicate the discrepancy. The modulation was halted after 50 cycles, with the effect of radiation damping evident at later times.
The main conclusion from Fig. 8 is that the shape oscillations appear to approach a steady state after an extended period, due to some unidentified limiting process. The simulation result shown here is independent of energy acceptance due to the relatively small oscillation amplitudes involved, ruling out bucket nonlinearity as a mechanism. The two major elements other than bucket nonlinearity that are present in the simulation but not in the theoretical result are radiation damping and quantum excitation. It is actually straightforward to add a damping term to the theory [28], the result being

$$
\begin{aligned}
\frac{\sigma^{2}(t)}{\sigma^{2}(0)} \cong & e^{-2 \beta t}\left[e^{2 \alpha t} \cos ^{2}\left(\Omega_{s 0} t+\pi / 4\right)\right. \\
& \left.+e^{-2 \alpha t} \sin ^{2}\left(\Omega_{s 0} t+\pi / 4\right)\right]
\end{aligned}
$$

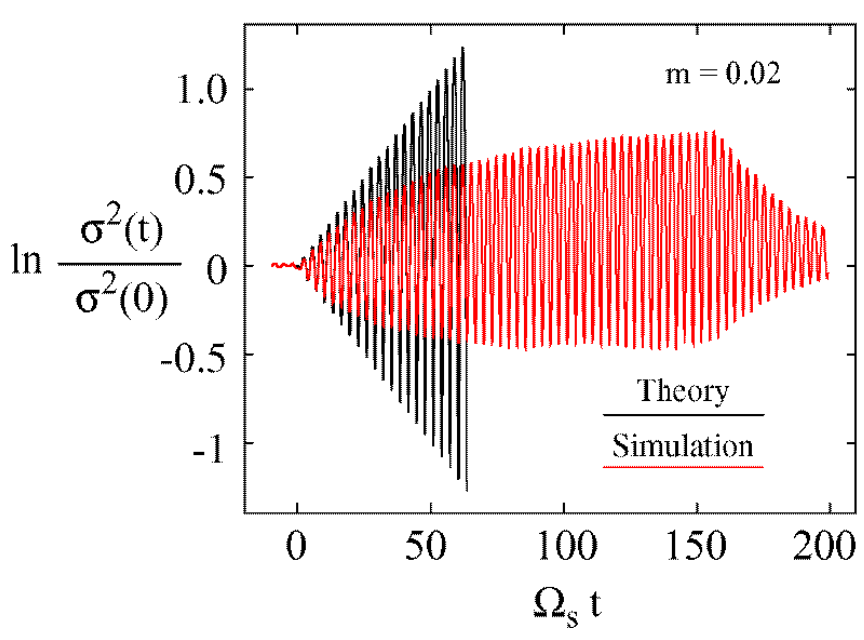

FIG. 8. (Color) Extended simulation result showing nonexponential behavior. 
where the radiation damping constant is $\beta=1 / \tau_{\varepsilon}$, the reciprocal of the longitudinal damping time. In addition, it is a simple matter to remove quantum excitation from the simulations while retaining radiation damping.

Shown in Fig. 9 is a comparison between Eq. (19) and a simulation result omitting quantum excitation, where the modulation constant $m$ was adjusted to cause the growth rate to cancel radiation damping, i.e., $\alpha=\beta$. In the simulation, the modulation was stopped after 20 cycles. From the excellent agreement between theory and simulation seen in the figure, it is clear that the primary cause of the discrepancy in Figs. 6 and 8 is quantum excitation.

As pointed out in Ref. [29], quantum excitation has a significant effect on longitudinal motion of individual particles in high-energy electron storage rings. As a result of intense quantum excitation, the intuitive notion of particles moving on closed paths in longitudinal phase space is only reliable for short periods of time. In the APS, quantum excitation causes the typical electron's momentum devia-

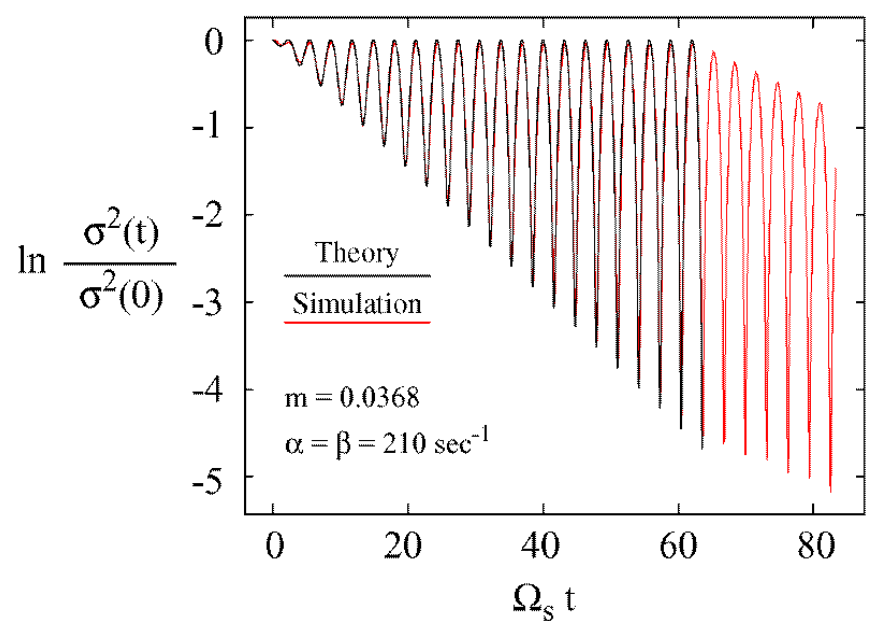

FIG. 9. (Color) Comparison between theory [Eq. (19)] and simulation, including radiation damping, but with no quantum excitation.

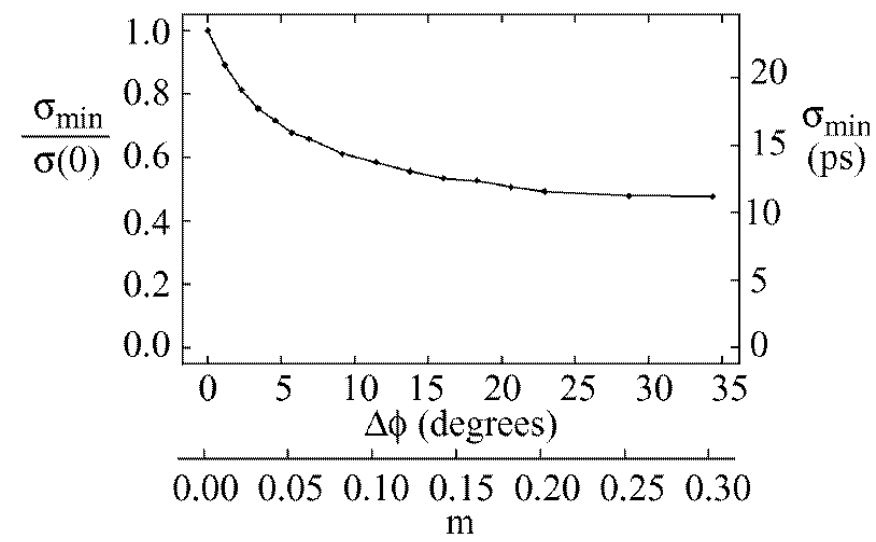

FIG. 10. Minimum bunch length vs phase-modulation amplitude from simulation. tion to change by about $\sigma_{\delta}(0.1 \%)$ in only 300 turns, which is about two synchrotron oscillation periods. Hence, it is not surprising that quantum excitation has a significant limiting effect on the response to the phase modulation.

Shown in Fig. 10 is the efficiency, from simulation, of bunch compression as a function of the phase-modulation amplitude for the APS storage ring. Both quantum excitation and bucket nonlinearity limit the attainable bunch length using this technique, although quantum excitation dominates.

\section{ENERGY SPREAD EFFECTS}

For bunch compression by a factor of 2 using this technique, the energy spread is approximately doubled, as indicated in Fig. 5. For insertion device (ID) beam lines, this impacts performance by both increasing the horizontal source size and broadening the undulator spectral lines, reducing brightness. In actual fact the energy spread at maximum compression for the strongest modulation is a bit more than doubled, but for the present discussion, a factor of 2 is a convenient factor to set the scale of the problem.

At the APS, the effective emittance for ID source points is $3.1 \mathrm{~nm}-\mathrm{rad}$, while the natural emittance is $2.5 \mathrm{~nm}$-rad. Effective emittance is the product of rms beam size and angular divergence at the ID source point, where the beam is focused to a waist. Nonzero dispersion at the ID source points $(17 \mathrm{~cm})$ together with the natural energy spread $(0.096 \% \mathrm{rms})$ increases the horizontal beam size from 221 microns rms to 276 microns - by the same factor relating natural to effective emittance. Doubling the energy spread results in an increase in the rms horizontal beam size by an additional $44 \%$, from 276 microns to $396 \mathrm{mi}-$ crons, resulting in an increase in effective emittance by the same factor. Since brightness is proportional to the reciprocal of emittance, this implies a 30\% loss in brightness associated with the increased beam size.

The second effect of increased energy spread is a broadening of the undulator spectral lines. This can be seen most easily from the formula for the on-axis radiated $x$-ray wavelength for undulator harmonic $n$ :

$$
\lambda_{n}=\frac{\lambda_{u}}{2 n \gamma^{2}}\left[1+K^{2} / 2\right] \text {. }
$$

Here $\lambda_{u}$ is the undulator period, equal to $3.3 \mathrm{~cm}$ for the standard APS undulator A design. The relativistic factor $\gamma$ is the particle's energy divided by its rest energy, and $K$ is the undulator strength parameter. Photon energy, proportional to the reciprocal of wavelength, thus scales as the square of beam energy, and an increase in energy spread translates directly into spectral line broadening and brightness reduction.

While detailed computations of the undulator spectrum, accounting for beam size, energy spread, and other factors 

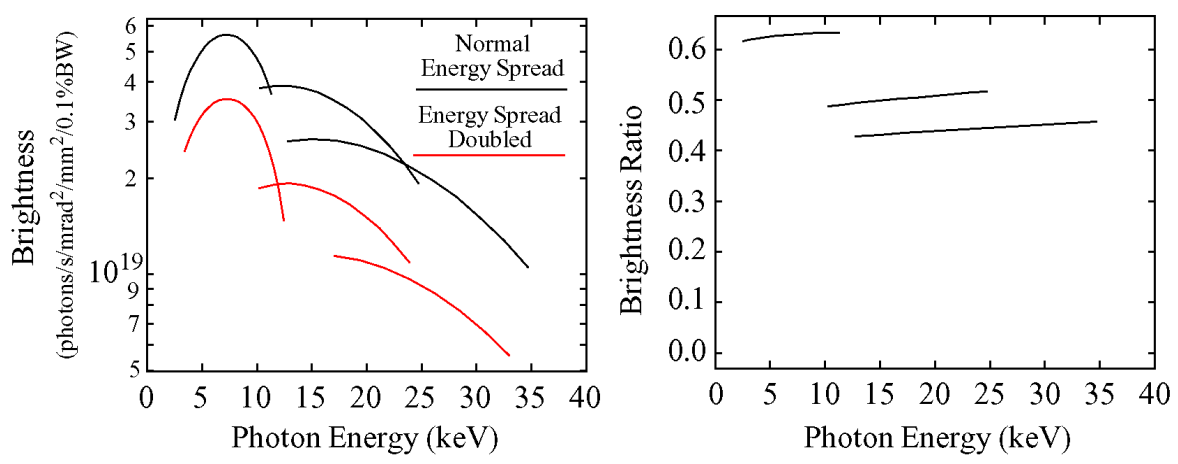

FIG. 11. (Color) Effect of increased energy spread on brightness for APS undulator A.

are somewhat involved, many computer codes are welldeveloped for computing on-axis brilliance [30,31]. Shown in Fig. 11 are tuning curves for APS undulator A, period = $3.3 \mathrm{~cm}, N_{\text {periods }}=72$, for the APS ring operating with $102 \mathrm{~mA}$ and $1 \%$ horizontal/vertical coupling. In the lefthand plot, the upper curves are for nominal APS operating conditions for the first, third, and fifth undulator harmonics, while the lower curves show the effect of doubling the particle beam's energy spread. The ratio of the two curves is shown in the right-hand plot, indicating that the overall loss of on-axis brilliance from energy spread effects lies in the range from $43 \%$ to $64 \%$. This includes both beam size and line broadening effects.

While the loss in brightness from energy spread effects is significant, it should be noted that the achievable duty cycle has a much more dramatic effect on the count rate for any x-ray experiment. For convenience, the experiment described below was conducted using the $2-\mathrm{Hz}$ APS injector repetition rate; however, the maximum possible rate is limited primarily by the time necessary for the beam to damp following removal of the excitation. Given the APS storage ring's 5-ms longitudinal damping time, this limiting rate is likely near $50 \mathrm{~Hz}$. A scheme to partially reverse the compression process by applying the modulation twice with a quarter-synchrotron period of delay between tone bursts may allow a somewhat higher repetition rate. In practice, many x-ray experimentalists interested in shortpulse operation conduct pump-probe experiments using a laser that is triggered near $1 \mathrm{kHz}$. Thus, the loss in number of usable photons is not as severe as one might think.

\section{EXPERIMENT}

To effect the required phase modulation, a special DC (i.e., low frequency) summing junction was inserted into the phase control regulation loop of one of the two APS rf systems, as shown in Fig. 12. An arbitrary function generator (AFG) was used to introduce a $3.6-\mathrm{kHz}$ phasemodulation tone burst at the APS injector $2-\mathrm{Hz}$ repetition rate (Stanford Research Systems DS345). A separate fast phase detector (Analog Devices AD 8302) was used to independently monitor the modulation, since the in-loop phase detector response demonstrated a roll off with increasing modulation frequency.

This phase control loop regulates the average phase of 8 out of the total of $16 \mathrm{rf}$ cavities installed in the ring. The second rf system was operated with no special hardware or control settings, but with an adjustable DC phase shift relative to the first system. Shown in Fig. 13 are oscilloscope waveforms characterizing the response of the first system to the application of eight cycles of $3.6 \mathrm{kHz}$ from the AFG. Other than startup shutdown transients, the phase tracks quite well. Eight cycles were chosen since the expected best compression from simulation occurs during the first few modulation cycles (Fig. 5), and beam loss will occur if strong modulation is applied for an extended period. The motivation for using a cosine wave for the simulations as shown in Fig. 4 instead of the sine wave of Fig. 13 was to allow direct comparison with the Mathieu equation in a standard form, as seen in Eq. (8). It is straightforward to modify the waveform used for the simulation to compare with experiment, which used sinusoidal waveforms.

After correction for the fast phase detector's DC offset, it is seen that using $150-\mathrm{mV}$ peak AFG drive voltage produced $\Delta \phi=19.5$ degrees peak of actual phase shift. Because of the in-loop phase detector's limited frequency response, a factor of 2 change in modulation frequency from 2 to $4 \mathrm{kHz}$, using the same drive voltage, resulted in over a factor of 3 increase in real phase-modulation amplitude.

A single bunch of approximately $5 \mathrm{~mA}(18 \mathrm{nC})$ was initially stored in the machine, and the total rf voltage was increased from the normal 9.4 MV to twice the synchrotron loss per turn, 10.8 MV. The relative phase between the two systems was shifted until the synchrotron tune arrived at the correct value, $1.8 \mathrm{kHz}$, assuring that the two systems were in quadrature. Klystron forward power was used as the best diagnostic to determine the correct sign of this phase shift. Since the on-crest system must provide the energy recovery function while the zero-crossing system performs no net work on the particle beam, the forward power must increase for the desired on-crest system and vice versa for the zero-crossing system as the relative phase 


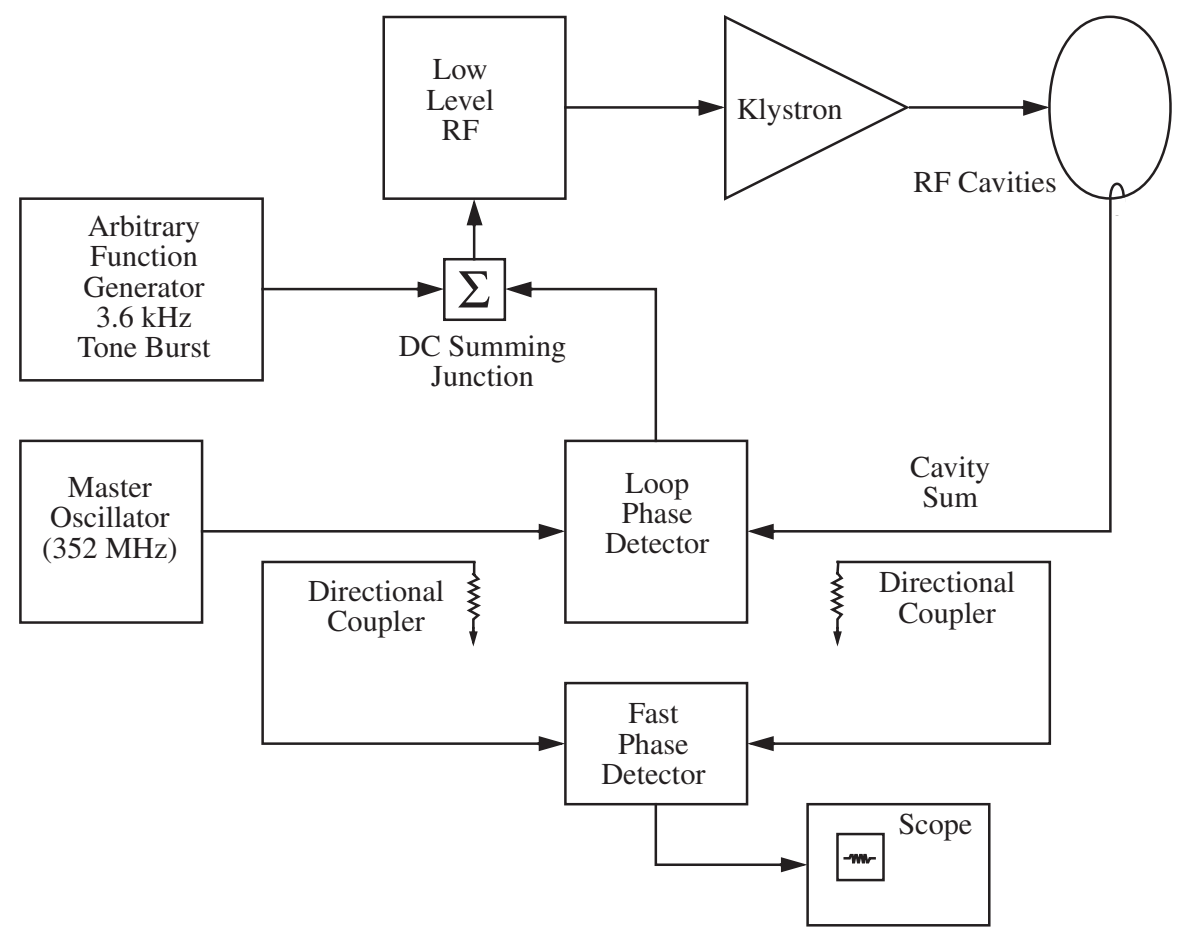

FIG. 12. rf phase control loop with phase modulation and additional monitoring.

between the two systems is moved in the correct direction. Note that the total stored beam current is limited by the available forward power from a single rf system. Up to $130 \mathrm{~mA}$ have been stored using two rf systems, limiting the total stored beam using this technique to about $65 \mathrm{~mA}$.
With four klystrons, up to $250 \mathrm{~mA}$ have been stored in the APS [32].

A Hamamatsu C5680 dual-axis streak camera installed in the visible light diagnostic bending magnet beam line at APS sector 35 was used to characterize the longitudinal
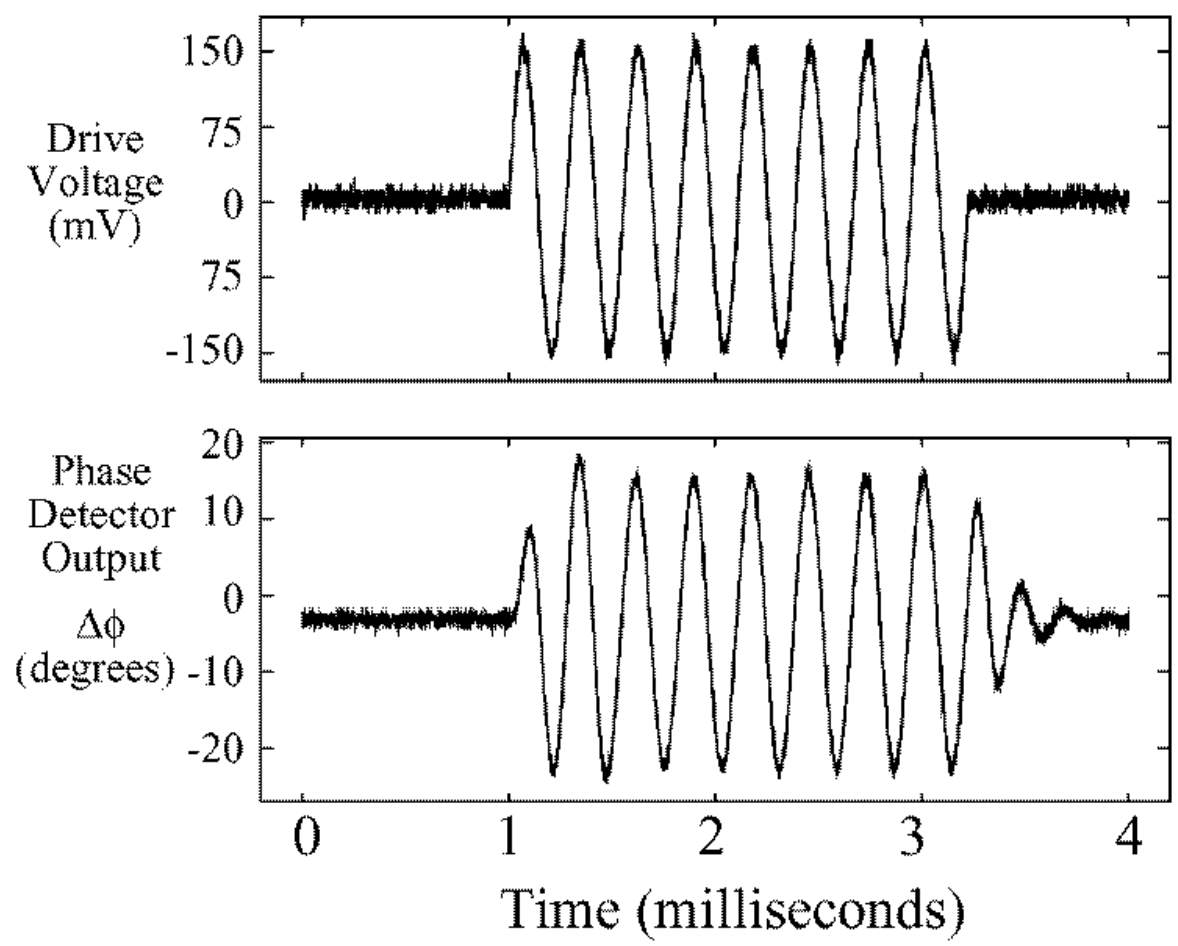

FIG. 13. Response of phase control loop to eight cycles of $3.6 \mathrm{kHz}$ from AFG. 

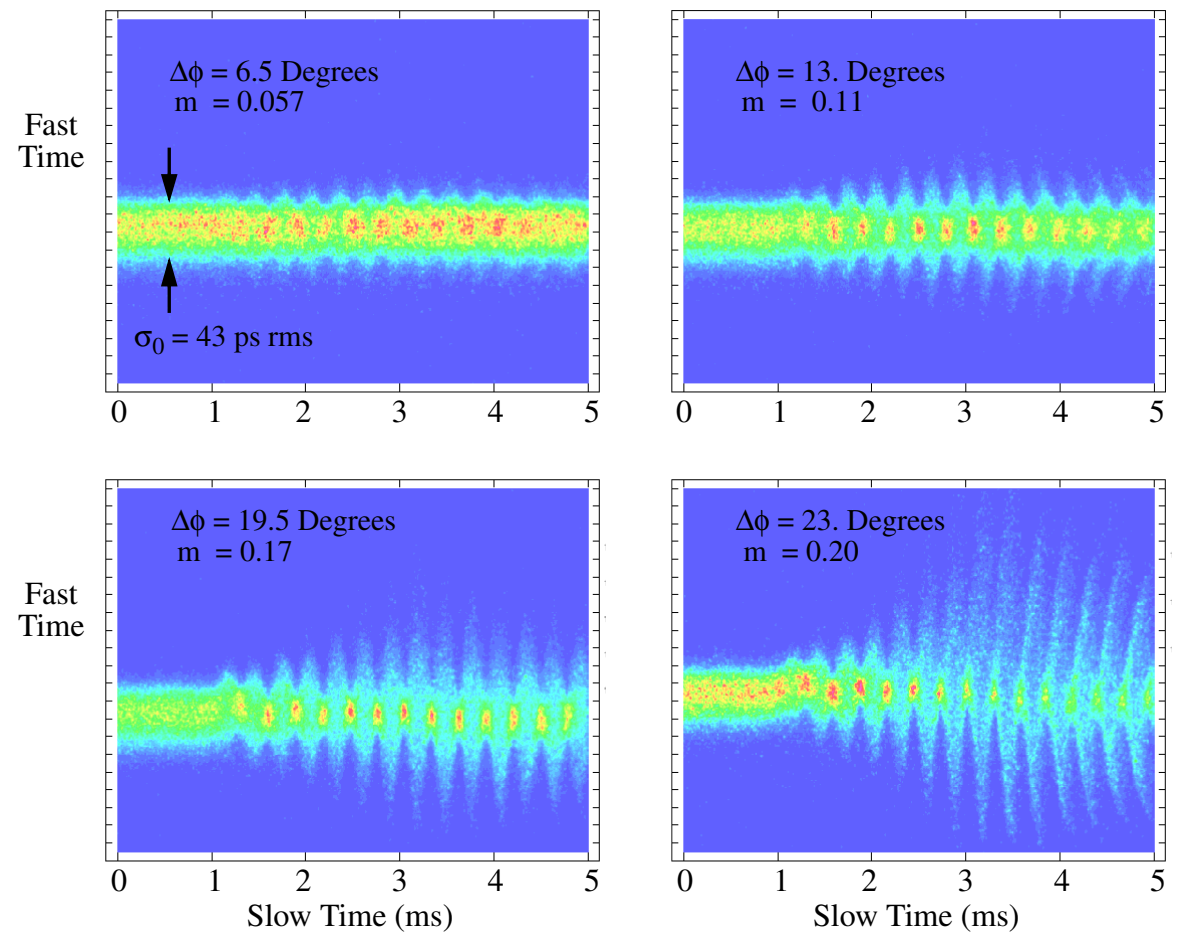

FIG. 14. (Color) Streak camera images showing effect of phase modulation.

shape oscillations [33,34]. A model M5675 synchroscan unit tuned at $117.3 \mathrm{MHz}$, one third of the master oscillator frequency, was used for the vertical sweep (fast time axis). The horizontal sweep unit, model 5679, provided selectable time ranges from $100 \mathrm{~ns}$ to $100 \mathrm{~ms}$. A 5-ms range was used for this experiment. Streak images were digitized by a DataCube MV200 video digitizer. The instrument has a bunch length resolution of $4 \mathrm{ps}$ rms on the sweep range used.

Figure 14 shows streak camera images collected using the phase modulation described by Fig. 13, as the phase-

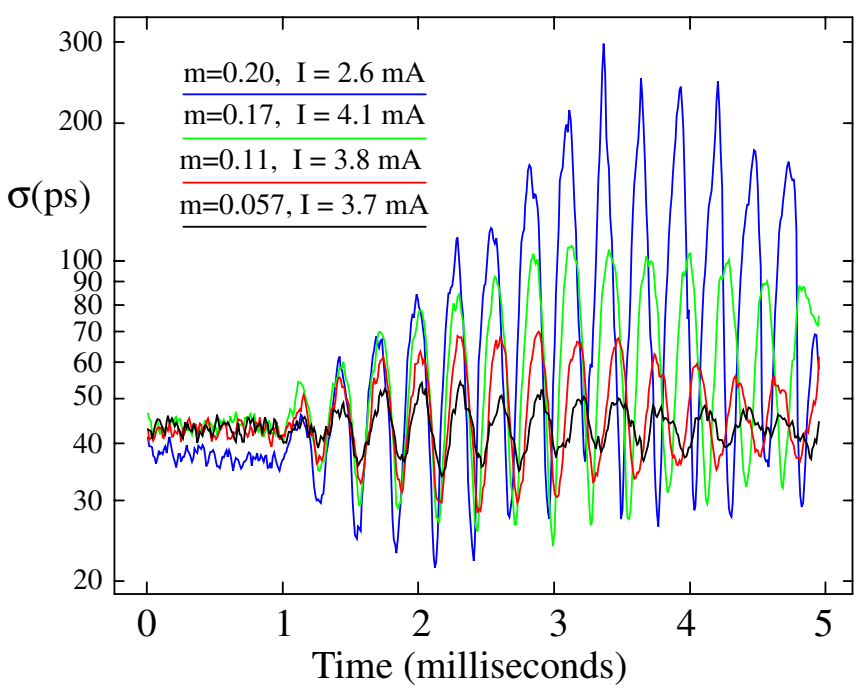

FIG. 15. (Color) Result of Gaussian fits of streak camera images. modulation amplitude was varied. Each image was sliced vertically and a Gaussian fit was made for each line to extract the rms bunch length as a function of time, shown in Fig. 15. The beam current stored is indicated, and is responsible for the variation seen in the initial bunch length. For the strongest modulation, the beam lifetime was reduced from 6 hours to 15 minutes, and corresponds to the last data set collected, with the lowest stored beam current and shortest bunch length observed.

In an effort to simulate the experimental conditions as closely as possible, a modulation waveform was constructed as the sum of 8 cycles of $3.6 \mathrm{kHz}$ with a small startup transient, empirically determined by subtracting the upper and lower waveforms in Fig. 13. The result is shown in Fig. 16. Simulation results are shown in Fig. 17.

While the minima seem to track reasonably well, the maximum bunch length from simulation is significantly larger than observed experimentally for all except the strongest modulation case, which was conducted with the smallest amount of stored beam. There is in addition an

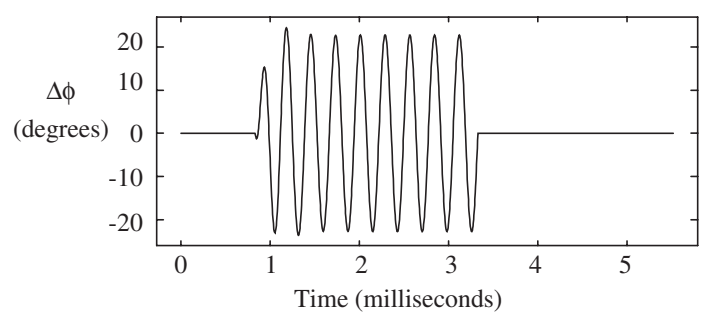

FIG. 16. Modulation waveform used for simulations. 

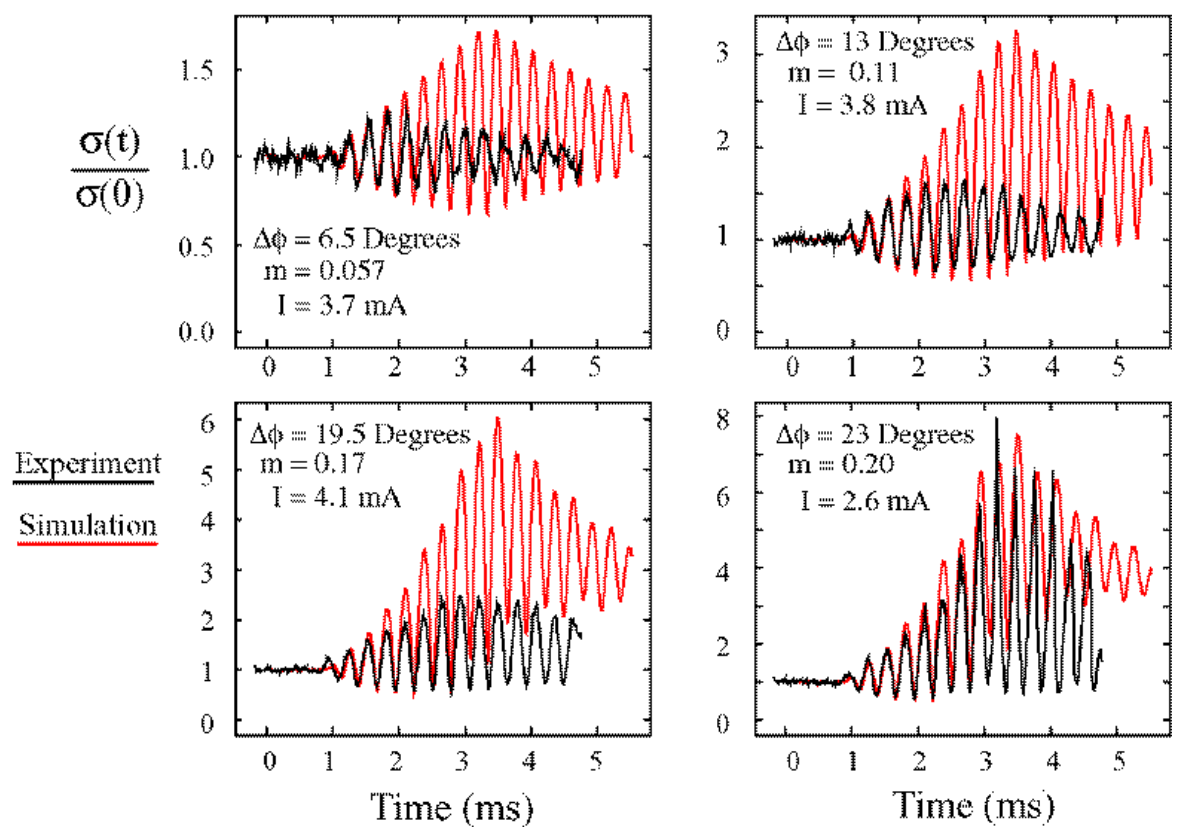

FIG. 17. (Color) Comparison between streak camera results and simulation.

apparent discrepancy in the frequency of the resulting shape oscillation, most apparent in the $m=0.11$ data. Variations in this frequency are even seen between the different experimental waveforms in Fig. 15. It is unlikely that the slow time base of the streak camera could vary by such a large amount over the course of the measurements, and oscilloscope measurements of the arbitrary function generator waveforms showed no evidence of a variation on this scale. It is certainly possible that these effects are partially the result of bunch-current-dependent collective effects, which were not included in the simulation. For example, potential well distortion tends to reduce the
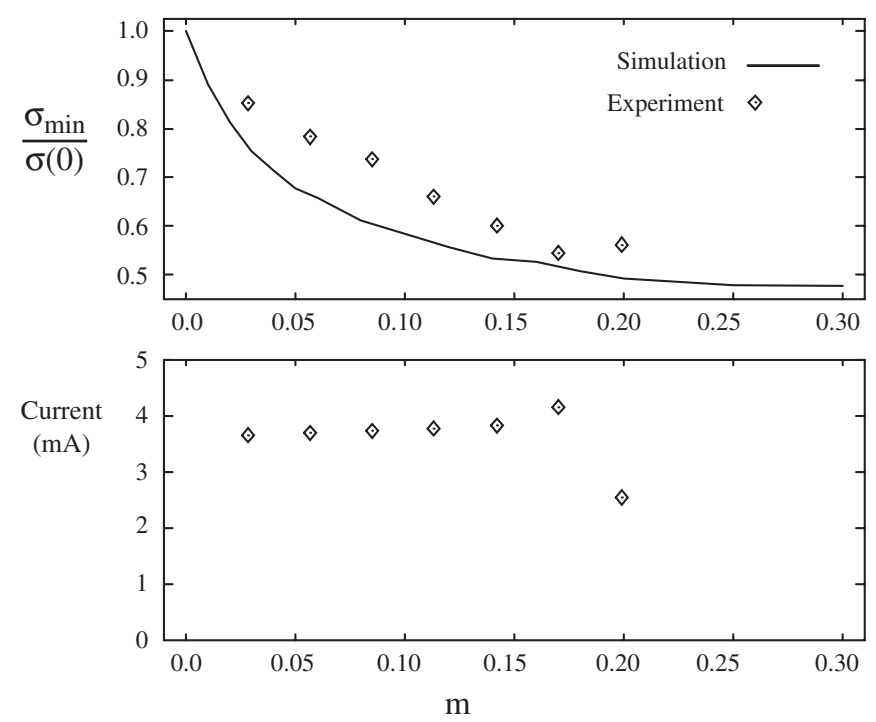

FIG. 18. Bunch compression efficiency and beam current vs modulation strength. synchrotron tune as the bunch current increases, which would bring the system out of resonance.

Finally, shown in Fig. 18 is a comparison between experimental and simulated bunch compression efficiency as a function of modulation strength $m$. Also shown are the bunch currents corresponding to each of the experimental data points. The shortest observed bunch length was $21.9 \mathrm{ps}$, occurring for the strongest modulation and lowest current. The fractional reduction in bunch length was greatest for slightly weaker modulation, with the largest beam current.

\section{CONCLUSIONS}

A method for transient bunch compression in highenergy electron storage rings using phase modulation has been modeled theoretically, simulated, and demonstrated experimentally. Compression by a factor of nearly 2 has been seen in the APS storage ring. Simulation results indicate that quantum excitation is the dominant factor limiting the compression process, with bucket nonlinearity playing a smaller role, at least for the APS. The best compression is seen during the first few cycles using the strongest allowable modulation amplitude.

While quantum excitation limits bunch compression to approximately a factor of 2 for the APS storage ring operating at $7 \mathrm{GeV}$, simulation results conducted at $5 \mathrm{GeV}$ indicate that compression by a factor of 3 should be possible. Because the energy loss per turn varies as the fourth power of energy, however, the on-crest rf system voltage must be reduced from $U_{0}=5.4 \mathrm{MV}$ to $1.6 \mathrm{MV}$ for $5-\mathrm{GeV}$ operation. At even lower beam energy, amplitude modulation is a more practical approach, since the on-crest 
voltage for the phase-modulation scheme becomes unreasonably small, and along with it the ability to modulate the synchrotron tune.

\section{ACKNOWLEDGMENTS}

This work was supported by the U.S. Department of Energy, Office of Science, Office of Basic Energy Sciences, under Contract No. W-31-109-ENG-38.

[1] John N. Galayda, SLAC-PUB-9847; http://www.slac. stanford.edu/pubs/slacpubs/9750/slac-pub-9847.pdf

[2] M. Cornacchia, J. Arthur, L. Bentson, R. Carr, P. Emma, J. Galayda, P. Krejcik, I. Lindau, J. Safranek, J. Schmerge, J. Stohr, R. Tatchyn, and A. Wootton, SLAC-PUB8950; http://www-ssrl.slac.stanford.edu/lcls/technotes/ 1cls-tn-01-7.pdf

[3] A. S. Schwarz, in Proceedings of the 2004 FEL Conference, Lucerne, 2004, p. 85.

[4] S. M. Gruner, D. Bilderback, I. Bazarov, K. Finkelstein, G. Krafft, L. Merminga, H. Padamsee, Q. Shen, C. Sinclair, and M. Tigner, Rev. Sci. Instrum. 73, 1402 (2002).

[5] C. Limborg, in Proceedings of the 1998 European Particle Accelerator Conference, Stockholm, 1998, p. 151.

[6] J. Feikes, K. Holldack, P. Kuske, and G. Wustefeld, in Proceedings of the 2004 European Particle Accelerator Conference, Lucerne, 2004, p. 1954.

[7] A. Zholents, P. Heimann, M. Zolotorev, and J. Byrd, Nucl. Instrum. Methods Phys. Res., Sect. A 425, 385 (1999).

[8] W. Guo, M. Borland, K. C. Harkay, V. Sajaev, and B. X. Yang, in Proceedings of the 2005 Particle Accelerator Conference, Knoxville, 2005, p. 3898.

[9] A. Drago, A. Gallo, A. Ghigo, M. Zobov, J. D. Fox, and D. Teytelman, Phys. Rev. ST Accel. Beams 6, 052801 (2003).

[10] S. Sakanaka and T. Obina, in Proceedings of the 2003 Particle Accelerator Conference, Portland, 2003, p. 3365.

[11] J. D. Fox and P. Corredoura, in Proceedings of the 1992 European Particle Accelerator Conference, Berlin, 1992, p. 1079.

[12] M. H. Wang, P. Chang, P. J. Chou, K. T. Hsu, C. C. Kuo, J.C. Lee, and W. K. Lau, in Proceedings of the 1999 Particle Accelerator Conference, New York, 1999, p. 2837.
[13] S. Sakanaka, M. Izawa, T. Mitsuhashi, and T. Takahashi, Phys. Rev. ST Accel. Beams 3, 050701 (2000).

[14] F. Orsini and A. Mosnier, Phys. Rev. E 61, 4431 (2000).

[15] P. Kuske, in Proceedings of the 1998 European Particle Accelerator Conference, Stockholm, 1998, p. 1297.

[16] Yu. Senichev, N. Hertel, S. Lunt, S. P. Moeller, and J. S. Nielsen, in Proceedings of the 1998 European Particle Accelerator Conference, Stockholm, 1998, p. 1339.

[17] S. Sakanaka, T. Mitsuhashi, T. Obina, and K. Umemori, in Ref. [8], p. 2887.

[18] J. M. Byrd, W.-H. Cheng, and F. Zimmermann, Phys. Rev. E 57, 4706 (1998).

[19] H. Huang et al., Phys. Rev. E 48, 4678 (1993).

[20] D. Li et al., Nucl. Instrum. Methods Phys. Res., Sect. A 364, 205 (1995).

[21] M. Bai, K. A. Brown, W. Fischer, T. Roser, N. Tsoupas, and J. van Zeijts, Phys. Rev. ST Accel. Beams 3, 064001 (2000).

[22] J. A. MacLachlan, Phys. Rev. ST Accel. Beams 4, 019001 (2001).

[23] E. Vogel, T. Bohl, and U. Wehrle, Phys. Rev. ST Accel. Beams 8, 102801 (2005).

[24] K. M. Fung, M. Ball, C. M. Chu, B. Hamilton, S. Y. Lee, and K. Y. Ng, Phys. Rev. ST Accel. Beams 3, 100101 (2000).

[25] H. Wiedemann, Particle Accelerator Physics I (SpringerVerlag, New York, 1999).

[26] A. Nayfeh, Introduction to Perturbation Techniques (Wiley Interscience, New York, 1981).

[27] M. Borland, Advanced Photon Source Light Source Note LS-287, 2000; http://www.aps.anl.gov/techpub/lsnotes/ 1s287.pdf

[28] E. Butikov, Eur. J. Phys. 25, 535 (2004).

[29] M. Borland, Phys. Rev. ST Accel. Beams 8, 074001 (2005).

[30] M. Sanchez del Rio and R. J. Dejus, in Proceedings of the Eighth International Conference on Synchrotron Radiation Instrumentation, San Francisco, CA [AIP Conf. Proc. 705, 784 (2004)].

[31] R. P. Walker and B. Diviacco, Rev. Sci. Instrum. 63, 392 (1992).

[32] A. Nassiri (Advanced Photon Source RF Group) (private communication).

[33] B.X. Yang and A. H. Lumpkin, in Ref. [8], p. 74.

[34] A. H. Lumpkin, F. Sakamoto, and B. X. Yang, in Ref. [8], p. 4185 . 